\title{
Proximity in Industry-Academia Collaborations: The Case of the ECHORD Project
}

\author{
Sascha Griffiths, Laura Voss and Florian Röhrbein \\ Technische Universität München, Robotics and Embedded Systems, Munich, Germany \\ Email: $\{$ sascha.griffiths, florian.roehrbein, laura.voss\}@in.tum.de
}

\begin{abstract}
In this contribution the authors examine robotics research itself. We look at the data which the European funded ECHORD project (European Clearing House for Open Robotics Development) generated. The project began in January 2009 with the ambitious goal of bringing together European robotics manufacturers with the European research institutions and it ended in 2013 . We will especially look at the effects of physical, geographical distance between partners in each of the sub-projects which are called experiments in the ECHORD terminology. We take dissemination activities and reporting outcomes as the measures of performance. Judging from the statistics we will show that collaborations across country borders show positive outcomes with regard to project outcomes. This shows that European robotics projects do indeed perform well if several partners from more than one European country are involved. ${ }^{1}$
\end{abstract}

Index Terms - university-industry, knowledge transfer, geographical proximity, robotics and automation

\section{INTRODUCTION}

It is often useful to look at the context in which artificial intelligence research takes place. This allows insights into how research can be made more effective (see for example [1, 2, 3]). In this contribution we look at the robotics research context which the ECHORD project provided. The technical results have already been presented elsewhere [4]. The project itself has also been subject to analysis previously [3, 5, 6, 7].

Robots may be one of the most significant applications for artificial intelligence in the coming years [8]. In 2004 Rodney Brooks [9] predicted that robots would be as ubiquitous as electronic mail and the World Wide Web by approximately 2019 . In 2013 , one can already see a trend towards this vision becoming a reality as robots already found commercial applications in entertainment, military applications, household and industrial service robotics, construction and heavy industry [10].

Overall, artificial intelligence has found many modern commercial applications [11]. This is in part due to an influx of venture capital which entered the scene in the 1970s [12]. Many see the influx of capital from largest private companies in recent times as a similar development in robotics [13].

Manuscript received Mar 15, 2014; revised Mar 30, 2014; accepted April 10, 2014.

Corresponding author: S. Griffiths (sascha.griffiths@in.tum.de)
However, a general delay between new scientific breakthroughs and their deployment in industrial and other commercial contexts has always been visible in robotics [14]. This is in part due to different cultures; whereas Japan has adopted robotics technology early, the USA has been more hesitant [15]. Also, the history of cooperations between academia and the industry has not always been easy due to the problem of finding common ground [16]. Often the industry is interested in profit which needs to be shown in the short term whereas research institutions take a long term view [17]. The fastest way to transfer technology is probably through direct cooperations [18]. Here we want to look at the conditions under which such efforts are fruitful. We are especially interested in whether dissemination activities are related to the distance between partners in international industryacademia collaborations.

\section{HELPFUL THEORETICAL AND EMPIRICAL BACKGROUND}

Academia-industry partnerships form one element of the National Innovation Systems [1, 19, 20]. These are the flows of technology and information among people, enterprises and institutions which are key to the innovative process on the national level [20]. However, within a European context they have to be contrasted with innovation systems which are more general flows of technology and information among people.

Especially in the recent European Union context the term National Innovation System needs to be reexamined in a transnational context. One needs to look at what impact transnational European projects have on sharing knowledge and advancing technology. The research and development (R\&D) activities which are funded by public investment through European Union grants need to be inspected as a means of improving the innovation systems within the EU. One of the ancillary questions is whether investment of public money is worth the costs if the effect is dwarfed by the barriers which may arise from the distances between collaboration partners on the European continent. The distances may be physical but these kinds of collaboration also face political, economic, cultural and linguistic barriers.

It has often been proposed [1] that innovation systems are comprised of codified knowledge which is being shared and tacit knowledge also named spillovers - which is harder to share. Ref. [21] also says that the distinction between codified and tacit knowledge often coincides 
with the distinction between knowing what (knowledge about the world) and know-how (competence). The latter is crucial for technology transfer were the relevant aspect is the transfer of competencies together with concepts of procedures. In this context one has to think about the feasibility of sharing tacit knowledge amongst project partners which are operating within a dispersed network which is spread over geographically long distances.

In the pertinent literature on knowledge sharing in academia-industry collaborations the role of geographical proximity is discussed controversially [22, 23, 24, 25, 26] Some argue that tacit knowledge is harder to share in contexts where physical proximity is high. Others argue that proximity effects are counteracted by modern information technology.

The measures that Arundel and Geuna [27] describe from their literature review for successful knowledge transfer include: scientific papers, citations of published papers, patents registered, patent citations and product announcements. For all these different effects are discussed with reference to the pertinent literature. For example, one can correlate the numbers of patents applied for or the number of product announcements which in this case serve as a proxy for innovative output - with the explanatory variables private and public spending (on the R\&D tasks). This yields results which show a positive effect of public investment on the innovation potential of R\&D activities. Using patents as a measure is however noted to be problematic [21] for various reasons (they cannot be used for inferences regarding the knowledge flow between industry and academia). Generally, all the measures listed above are codified knowledge and it is harder to tackle the issues connected to tacit knowledge. This is almost a definitional point. The main correlate of successful transfer in R \& D from academia to industry is probably products which emerge from collaborations. However often these become available after the project ends and are therefore not useful as an indicator for the monitoring of ongoing knowledge transfer initiatives. Therefore, it is easier to track the project progress and the output as codified knowledge during a projects run time.

\section{THE ECHORD PROJECT DATA}

The idea of the ECHORD project was born before the economic crisis had its maximum impact on the robotics industry in 2008/2009. Therefore, the concept of a project with the clear goal to strengthen the collaboration between academia and industry was a good opportunity to support the industry by offering funding opportunities and fostering already existing networks and creating new partnerships with the academic world ${ }^{2}$.

The ECHORD concept comprises two instruments to reach the overall goal: the first one is the funding of socalled experiments, small research and development projects, carried out typically by 2-3 partners, both from industry and academia.

\footnotetext{
${ }^{2}$ The project itself was proposed as an Integrating Project (IP) in the Call ICT-2007.2.2 under the 'Challenge 2: Cognitive Systems, Interaction, Robotics'.
}

The second instrument is the so-called structured dialogue, a means to strengthen the relationship between academic institutions and industrial companies, and to identify and support the knowledge transfer.

Out of the 51 sub-projects $17(27.45 \%)$ are of the category joint enabling technology, $20(39.21 \%)$ are aimed at application development and $14(33.33 \%)$ are feasibility studies. However, these categories should not been seen as mutually exclusive. The applied nature of the project is underlined by the foci of these activity types.

For the purpose of examining proximity effects, the bimonthly reports, the final reports and review reports of all 51 experiments which had ended by September 2013 have been examined in detail. The data contained in these sources were quantified for the purpose of this investigation.

What we wanted to examine here is whether a long distance between two or more experimenting partners shows a detrimental effect on the experiment outcome. Further, a closer look will be taken at the effect of national borders on the experiments' outcomes.

We employ four measures as performance indicators for the experiments.

- Number of dissemination activities.

- A measure of conforming to the proposed time plan.

- A measure of project success in terms of receiving flags from independent reviewers

- A measure of project success in terms of achieved project objectives as rated by independent reviewers

All four of these types of measures can be seen as operationalization of progress within an experiments execution. One measures the codification of knowledge in the various formats of dissemination activities as listed below. The others try to grasp the progress at run time and the technological advances after the project has ended.

Information extracted from the final reports relates to their dissemination activities. The information is of course self-reported and should be taken as such. The categories of dissemination activities are diverse: websites, talks, student activities, conference presentation, paper (conference or workshop), conference poster, organization (e.g. workshop), tutorial, public event/trade fair, media and TV, competition, journal paper, journal special issue, YouTube video, other. These are all methods which the project chose to employ to communicate the knowledge gain throughout the runtime of their individual experiments.

Additionally, the experiments reported on a bimonthly basis on their activities and a moderator judged their progress via a traffic light system. A green traffic light means that there are no deviations from the experiment plan. A yellow traffic light means that there are possible delays. A red traffic light judges the experiment to be delayed. This information has been used to generate a second measure of success for the Experiment.

The review reports were also examined. Independent experts rated the experiments' performance. There were two quantifiable aspects of these reports. The experts 
rated whether the experiments were flagged for particular success in a given area. Also, the experiments were judged as to whether they were to be considered to have achieved their objectives.

Also, for the purpose of this study experiments were split up into categories. First, we distinguish between experiments which involved only one partner (thus not crossing a country border) and those which involved partners from more than one country (thus crossing at least one country border). The difference between the two categories with respect to the distributions of experiments according to the categories is shown in Fig. 1.

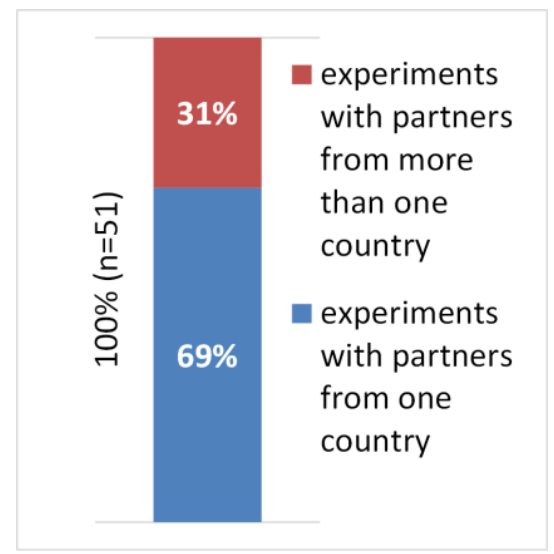

Figure 1: Percentage of ECHORD experiments classified according to whether the collaboration partners where located within the same country or in more than one country. The experiments which had partners from only one country involved are on the left. Those which involved partners from more than one country are on the right.

Second, we distinguished between those experiments which had a lower physical, geographical distance between the partners than the overall mean of all distances between partners and those which had a larger physical, geographical distance between the partners. Out of all 51 experiments the majority $(51 \%)$ had a distance great than $138 \mathrm{~km}$, which is the median for all experiment distances. The minority (49\%) was in the category of having less than that distance between its partners (see Fig. 2).

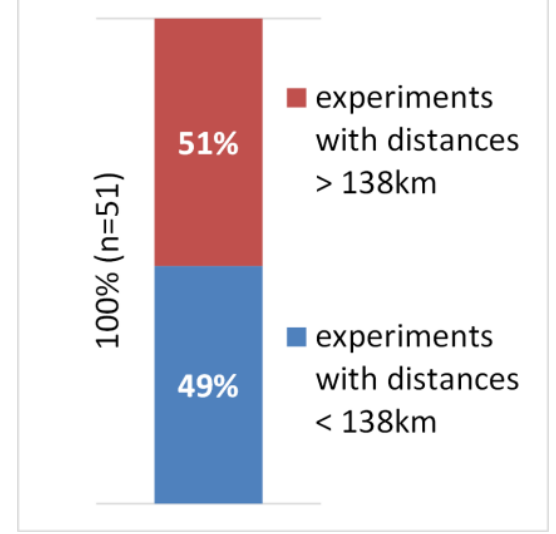

Figure 2: Percentage of ECHORD experiments classified according to the physical, geographical distance between partners. The experiments which had a distance smaller than the median are on the left. Those with a distance greater than the median are on the right.
Classifying the experiments in terms of these properties of the partnerships shows a first result which respect to the structure of collaborations. The experimenting partners more frequently collaborated at longer distances but within their own country than collaborating across a country border. The short and long distances are almost balanced whereas one can see a clear preference for collaborations within the same country.

\section{RESUlTs}

For each of the four performance indicators we looked at whether there was a noticeable effect of collaboration across country boarders or with respect to geographical distance between the partners. We first examined the descriptive statistics and then performed a $\chi^{2}$-test to determine whether the observations are indeed significant.

The literature predicts that in such situation where the direct face to face communication of knowledge is harder, more knowledge needs to be codified. This means that each partner in projects with little physical proximity will need to find means for communicating their results to the other partner more than projects which have a direct physical proximity. This leads to the hypothesis in this specific case that an ECHORD experiment in which the partners are further apart within Europe with respect to their physical locations will be more active with regard to communicating their results. This is can be tested on the data at hand.

The dissemination activities can be grouped into those which are peer-reviewed publications and those which are more general dissemination activities (see above for details). The experiments which involved partners from only one country published slightly less peer-reviewed publications on average $(M=2.11, S D=3.27)$ than those which involved partners from more than one country $(M$ $=2.18, S D=2.99$ ). However, the difference is minute and does not indicate a great difference between the two groups of experiments. Though, the standard deviation indicates more variability in the set of experiments involving partners from only one country. This is due to the minimum of peer-reviewed papers being equal at 0 for both groups with some partners producing no scientific papers and the maximum being 17 for experiments with partners from only one country and 9 for those with partners from only one country.

For other dissemination activities the number was almost equal for experiments which involved partners from only one country $(M=7, S D=5.48)$ and those which those which involved partners from more than one country $(M=7.06, S D=6.25)$. The minimum for the former was 1 while the latter produced at least 2 other dissemination activities. This explains the slightly larger standard deviation. Thus, one both measures of dissemination activities one can see no great differences meaning that country borders have no effect negative or positive on the experiment performance in this area in our data set.

The traffic light system which ECHORD employed indicated whether a project was on time or not. A moderator nominated by the core consortium of the project read bi-monthly reports and could either give a green traffic 
light if the project was on schedule or a different color if the experiment had fallen behind their plan.

The experiments which involved partners from only one country performed slightly worse with respect to green traffic lights $(M=5.35, S D=3.00)$ than those which involved partners from more than one country $(M$ $=6.06, S D=3.47)$. The absolute minimum of 0 was reached in the former category, meaning at least one experiment failed to be on time with any report and the maximum reached was 10 . The latter had a minimum of 1 and a maximum of 12 . This indicates that collaborations across country borders stay on time more than those which have closer proximity.

Checking whether the opposite is also true, one realizes that the hypothesis does not hold. Within country collaborations were penalized less often $(M=7.37, S D=$ 4.10) than those which involved partners from more than one country $(M=8.25, S D=5.29)$. The second set produces greater variability with the first group having a range of 0 to 19 and the second having a range of 1 to 18 . This means that overall there were more delay ratings for the second group, too.

The independent experts who reviewed the experiments judged experiments assigning flags for particularly notable achievements in eights pre-defined categories. The notable results is that experiments which involved partners from only one country performed worse with respect to flags $(M=0.82, S D=1.12)$ with an average below even one flag. On the contrary, experiments which involved partners from more than one country performed twice as good on average $(M=1.68, S D=1.86)$. The absolute minimum of 0 was reached in the former category, meaning at least one experiment failed to receive any flag and the maximum reached was 4 flags. The latter had a minimum of 0 but at least one experiment received 6 flags out of eight. This indicates that collaborations across country borders where on average rated better by the reviewers than those which only had partners in the same country.

Reaching objectives, which was also judged by the reviewers of the experiments, needed to be quantified slightly differently as the categories were "yes", "partial" and "no". We can see here that $54 \%$ out of the onecountry category reached a positive evaluation whereas $50 \%$ of the experiments in the more-than-one-country category received a "yes". The "partial" and "no" verdict taken together was given to $46 \%$ out of the experiments in the former category whereas $50 \%$ out of the latter category received either of these evaluations. We can therefore see here that the category of experiments which only collaborated within one country was slightly better at achieving their agreed upon results.

The data can also be examined with regard to the same performance indicators but with the experiments grouped according to their physical, geographical distance between the partners.

The experiments which involved partners which were less than $138 \mathrm{~km}$ apart published fewer peer-reviewed publications on average $(M=1.96, S D=3.51)$ than those which involved partners for which the distance was great than $138 \mathrm{~km}(M=2.30, S D=2.85)$. However, the former group showed a greater variance in their publication behavior. This is due to the minimum of peer-reviewed papers being equal at 0 for both groups with some partners producing no scientific papers and the maximum being 17 for experiments with a smaller distance and 9 for those with a greater distance.

For other dissemination activities the number was almost equal for experiments which involved partners with a geographical distance smaller than $138 \mathrm{~km}(M=7, S D$ $=6.91$ ) and those which involved partners that were further apart than the median $(M=7.19, S D=5.9)$. The minimum was 1 other dissemination activity for both groups. Whereas in the former group at least one experiment produced 29 such activities the latter had only 25 as its maximum. It can thus be stated that on average there is not a large difference in dissemination activities between the groups. Although, the greater distance group will produce slightly more peer-reviewed papers the other group has performed slightly better with regard to other dissemination activities.

The traffic light system shows a slight disadvantage for the smaller distance. The experiments which involved partners with a geographical distance smaller than 138 $\mathrm{km}$ performed slightly worse with respect to green traffic lights $(M=4.84, S D=2.91)$ than those for which the distance was greater than the median of all experiments $(M=6.15, S D=3.16)$. The absolute minimum of 0 was reached in the former category, meaning at least one experiment failed to be on time with any report and the maximum reached was 10 . The latter had a minimum of 1 and a maximum of 12. This indicates that collaborations across country a greater physical distance stay on time more than those which have closer proximity.

Checking whether the opposite is also true, one recognizes that the hypothesis does not hold. Experiments within the lower distance group were penalized less often $(M=6.80, S D=4.28)$ than the greater distance group $(M$ $=8.46, S D=4.41)$. The second set produces great variability with the first group having a range of 0 to 19 and the second having a range of 1 to 18 . This means that overall; there were more delay ratings for the second group, too. This mirrors the results for the comparison for within country and across border collaborations closely.

The independent experts who reviewed the experiments judged experiments which collaborated across a smaller distance slightly worse with respect to flags ( $M=$ $0.88, S D=1.16$ ) with an average below even one flag and a maximum of 4 flags. On the contrary, experiments which collaborated across a larger distance performed better on average $(M=1.30, S D=1.64)$. The absolute minimum of 0 was reached in the former category, too, whereas the maximum was 6 flags. This again shows that collaborations across a larger distance can be successful.

One can see here that that $52 \%$ out of the low distance category reached a positive evaluation whereas $54 \%$ out of the higher distance experiments category received a "yes". The "partial" and "no" verdict taken together was given to $48 \%$ of experiments in the former category whereas $46 \%$ out of the latter category received either of 
these evaluations. We can therefore surmise here that a greater distance was actually not detrimental to the project outcome in our data set.

Chi-square tests of independence were performed to examine the relation between (a) the number of countries involved in an experiment and (b) the geographical distance between the partners in an experiment, and experiment success, as indicated by the different variables.

Experiment success, measured as the number of flags per experiment, was significantly influenced by the number of countries involved (1 country vs. more than 1 country; $\left.\chi^{2}(1, N=51)=7.67, p<0.01\right)$

However, the number of countries involved did not influence the color of traffic lights awarded to the experiments $\left(\chi^{2}(1, N=51)=0.01, p=0.92\right)$, the dissemination activity of the experiments $\left(\chi^{2}(1, N=51)=0\right.$, $p=1$ ), or the successful achievement of the experiments' objectives $\left(\chi^{2}(1, N=51)=0, p=1\right)$.

Geographical distance had, on the other hand, a significant influence on the successful achievement of the experiments' objectives $\left.\left(\chi^{2}(1, N=51)=0.02, p<.05\right)\right)$.

It did not influence the number of flags $\left(\chi^{2}(1, N=\right.$ $51)=2.03, p=0.15)$, color of traffic lights $\left(\chi^{2}(1, N=51)=0, p=1\right)$, or the dissemination activity of the experiments $\left(\chi^{2}(1, N=51)=0.16, p=\right.$ 0.69).

\section{DISCUSSION}

The data analysis above has provided some insights as to what effects of physical distance are to be expected in collaborative robotics projects. This pertains to the geographical distance as well as separation by a country border.

The four different performance indicators (dissemination activities, traffic lights, flags and objectives) show different interactions with the two distance variables.

What can be deduced is that there are no discernable effects of the existence of a national border or geographical distance between the partners on dissemination activities. This is contrary to the predictions found in the literature. Possibly, use of modern information technology mitigates the effects found in the literature previously.

Further, we have demonstrated that the moderator evaluations of projects keeping to their time plan could in principle be interpreted either way depending on whether the positive or the negative feedback is considered. An interpretation is hard to make as the results for this variable were not significant. However, one can infer that there are no detectable negative outcomes of long distance or international collaborations during the run-time of such projects.

Although the difference seems small, national borders had a significant effect on the number of flags experiments received. Here international collaborations performed slightly better than within country collaborations. In terms of effects of distance between partners no concrete result can be presented. But the higher average number of flags again speaks for long distance collaborations.
In terms of achieving objectives, collaborations within a single country seem to be at a slight advantage judging by the ECHORD data. Though, the difference is not significant. In contrast, there was a significant result for long distance collaborations performing better in terms of achieving objectives.

The results suggest that there are at least few disadvantages for international and long distance collaborations in robotics projects.

\section{CONCLUSION}

The results presented here stem from a large robotics project with over a hundred partners. We aimed at testing whether international and long distance collaborations in research and development are actually efficient. We have presented our findings which suggest that actually physical distance has more positive than negative effects on project outcomes.

\section{ACKNOWLEDGMENT}

Our research has been supported by the European Union through the 7th Framework Programme (FP7) under grant agreement number FP7-ICT-231143.

\section{REFERENCES}

[1] G. Myers, "Politeness and Certainty: The Language of Collaboration in an Al Project," Social Studies of Science, vol. 21, no. 1, pp. 37-73, 1991.

[2] E. Reiter, "The shrinking horizons of computational linguistics," Computational Linguistics, vol. 33, no. 2, pp. 283-287, 2007.

[3] G. Veiga, C. Silva, R. Araujo, N. Pires, and B. Siciliano, 'The ECHORD project proposals analysis-research profiles, collaboration patterns and research topic trends', Expert Systems with Applications, vol. 40, no. 17, pp. 7132-7140, 2013.

[4] F. Röhrbein, G. Veiga, and C. Natale, "Gearing Up and Accelerating Cross-fertilization between Academic and Industrial Robotics Research in Europe: Technology Transfer Experiments from the ECHORD Project," Springer: Heidelberg, 2014.

[5] F. Röhrbein, S. Griffiths, and L. Voss, "On IndustryAcademia Collaborations in Robotics," Technical Report, TUM-I1338, 2013.

[6] S. Griffiths, C. Natale, R. Araujo, G. Veiga, P. Chiacchio, F. Röhrbein, S. Chiaverini, and R. Lafrenz, "The ECHORD project: A general perspective," in Gearing up and accelerating cross-fertilization between academic and industrial robotics research in Europe, F. Röhrbein, G. Veiga, C. Natale, Eds., Heidelberg: Springer, 2014, pp. 124.

[7] S. Griffiths, L. Voss, and F. Röhrbein, "IndustryAcademia Collaborations in Robotics: Comparing Asia, Europe and North-America," in IEEE International Conference on Robotics and Automation (ICRA 2014), in press.

[8] R. Bogue, "The role of artificial intelligence in robotics," Industrial Robot: An International Journal, vol. 41, no. 2, pp. 2-2, 2014.

[9] R. Brooks, "The robots are here," Technology Review, vol. 107, no. 1, pp. 30-30, 2004. 
[10] G. A. Bekey, Autonomous Robots, Cambridge, MA: MIT Press, 2005.

[11] K. D. Forbus, "AI and Cognitive Science: The Past and Next 30Years," Topics in Cognitive Science, vol. 2, no. 3, pp. 345-356, 2010

[12] L. Steels, "Fifty years of AI: From symbols to embodiment-and back," in 50 years of artificial intelligence, M. Lungarella, F. Iida, J. Bongard, and R. Pfeifer, Eds. Berlin / Heidelberg: Springer, 2007, pp. 18-28.

[13] F. Tobe. (2003). "Apple, Amazon and now Google: It's a pretty exciting day for robotics!" [Online]. Available: http://robohub.org/apple-amazonand-now-google-its-apretty-exciting-day-forrobotics

[14] U. Schmoch, "Double-boom cycles and the comeback of science push and market-pull," Research Policy, vol. 36, no. 7, pp. 1000-1015, 2007.

[15] S. J. Shim and A. Kumar, "Development of the robotics industry in the United States and Japan: a comparison of competitive factors," Competitiveness Review: An International Business Journal incorporating Journal of Global Competitiveness, vol. 16, no. 1, pp. 57-66, 2006.

[16] R. Lafrenz, "Rise of the robots," Public Service Review: European Union, vol. 18, 2009.

[17] F. Röhrbein and S. Griffiths. (2013). "Academic and industry collaboration (Part 3): Interview with Minoru Asada." [Online]. Available: http://robohub.org/academicand-industrycollaboration-part-3-interview-withminoruasada

[18] S. Griffiths and F. Röhrbein, "Technology Transfer in Academia-Industry Collaborations - Interview with Dr. Alexander Waibel," KI - Künstliche Intelligenz, vol. 27, no. 4, pp. 365-368, 2013.

[19] J. Fagerberg and M. Srholec, "National innovation systems, capabilities and economic development," Research Policy, vol. 37, pp. 1417-1435, 2008.

[20] OECD, “National Innovation Systems," Technical report, 1997.

[21] B. Johnson, E. Lorenz, and B.-A. Lundvall, "Why all this Fuss about Codified and Tacit Knowledge," Industrial and Corporate Change, vol. 11, pp. 245-262, 2002.

[22] R. Ponds, F. van Oort, and K. Frenken, "The geographical and institutional proximity of scientific collaboration networks," in ADRES International Conference 2006, vol. 86, 2006, pp. 423-443.

[23] R. Ponds, F. van Oort, and K. Frenken, "The geographical and institutional proximity of research collaboration," $\mathrm{Pa}$ pers in Regional Science, vol. 86, pp. 423-443, 2007.

[24] J. M. Rosa and P. Mohnen, "Knowledge Transfers between Canadian Business Enterprises and Universities: Does Distance Matter?," Annals of Economics and Statistics / Annales d'E'conomie et de Statistique, 2007, pp. 303-323.

[25] L. Biggiero and A. Sammarra, "Does geographical proximity enhance knowledge exchange? The case of the aerospace industrial cluster of central Italy," International
Journal of Technology Transfer \& Commercialization, vol. 9, no. 4, pp. 283-305, 2010.

[26] S. W. Cunningham and C. Werker, "Proximity and collaboration in European nanotechnology," Papers in Regional Science, vol. 91, no. 4, pp. 723-742, 2012.

[27] A. Arundel and A. Geuna, "Does proximity matter for knowledge transfer from public institutes and universities to firms?,", SPRU Working Paper, no. 73, 2001.

Sascha Griffiths is a research associate at the research group "Robotics and Embedded Systems" in the Informatics Department of Technische Universität München. Previously he held research positions at the University of Kent and Bielefeld University. He received an MA for Bielefeld University in 2006 and a PhD from the University of Kent in 2013.

Laura Voss graduated from Ludwig-Maximilians-Universität Mün-

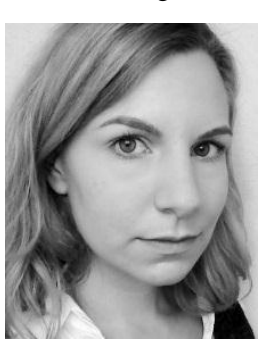
chen with a Diplom in organizational and neuro-cognitive psychology in 2010 . She is a research assistant in the research group "Robotics and Embedded Systems" at the Informatics Department of Technische Universität München, where she is involved in the project management and quality management of various EU-funded robotics research projects. She is a $\mathrm{PhD}$ student at the Chair of Sociology of Science (Technische Universität München) and the Munich Center for Technology in Society (MCTS).

Florian Röhrbein is a senior lecturer at the research group "Robotics

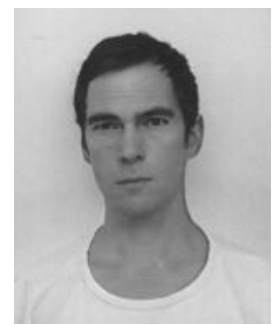
and Embedded Systems" in the Informatics Department of Technische Universität München. He was member of the Executive Committee of the ECHORD project and is now managing director of the Neurorobotics subproject of the Human Brain Project flagship. Research stays include the MacKay Institute of Communication and Neuroscience (UK), the HONDA Research Institute Europe (Germany) and the Albert Einstein College of Medicine (New York). He received his Diploma (with honors) and $\mathrm{PhD}$ (magna cum laude) from TU München. In 2011 he received the venia legendi for computer science from Universität Bremen. 